\title{
¿Son útiles las prótesis metálicas autoexpandibles en el control de la hemorragia digestiva varicosa refractaria?
}

\section{Are self-expanding metal prostheses useful for controlling refractory variceal digestive hemorrhaging?}

Reinaldo Andrés Rincón S. ${ }^{1}$

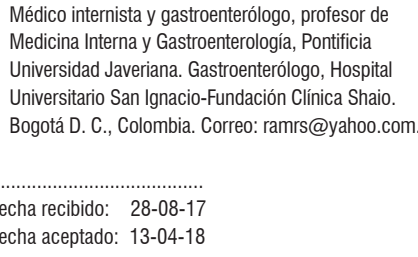

\begin{abstract}
Resumen
El sangrado varicoso es una urgencia médica con altas tasas de morbimortalidad. De los pacientes que llegan para recibir manejo hospitalario, hasta un $10 \%$ no responden a medidas hemostáticas convencionales, por lo que se ha planteado el uso de prótesis metálicas autoexpandibles para el manejo con resultados prometedores de dichos pacientes.

En el presente documento se discute la literatura mundial disponible acerca de la experiencia en el uso de dichos dispositivos; la eficacia en el control agudo; el pronóstico a largo plazo; el tipo de prótesis utilizadas y las alternativas, complicaciones y retos del manejo de pacientes con esta técnica, que puede considerarse emergente en nuestro medio.
\end{abstract}

\section{Palabras clave}

Sangrado varicoso refractario, terapia de rescate, prótesis metálicas autoexpandibles, manejo endoscópico.

\section{Abstract}

Variceal bleeding is a medical emergency which has high rates of morbidity and mortality. Up to $10 \%$ of these patients treated in hospitals do not respond to conventional hemostatic measures. For this reason, the use of self-expanding metal stents (SEMS) has been proposed for management of these patients. It has had promising results.

This paper discusses the literature available internationally about experience with the use of SEMS, their efficacy for acute control, long-term patient prognoses, types of stents, alternatives, complications, and challenges for the use of SEMS for patient management that should be considered in our environment.

\section{Keywords}

Refractory variceal bleeding, rescue therapy, self-expanding metal stents, endoscopic management.

\section{INTRODUCCIÓN}

La hemorragia digestiva por ruptura de várices esofágicas es una de las complicaciones más frecuentes y dramáticas asociadas con la cirrosis hepática. La incidencia de esta complicación llega al 25\% de los pacientes con hipertensión portal y tiene una mortalidad media a las 6 semanas superior al $20 \%$, se presenta con mayor frecuencia propor- cionalmente al deterioro en la función hepática ( $10 \%$ de los pacientes con Child-Pugh A y hasta el 70\% con Child-Pugh C). Otra característica de esta complicación es la elevada recidiva que se presenta en los primeros 2 a 3 meses posteriores al episodio hemorrágico $(1,2)$.

Teniendo en cuenta el panorama dramático que propone un episodio de sangrado varicoso, es de vital importancia desarrollar estrategias dirigidas a prevenir el primer epi- 
sodio de sangrado y evitar su recidiva. Uno de los eventos más importantes es poder identificar a los pacientes que van a sangrar. Los factores de riesgo asociados con ruptura varicosa son múltiples e incluyen presión portal y varicosa; características endoscópicas de las várices, especialmente el tamaño y la presencia de signos rojos; y severidad de la hepatopatía y su etiología.

Infortunadamente, en muchos casos hay fallas en el control de factores de riesgo y se desencadena un episodio de sangrado digestivo varicoso, que en un $15 \%$ es de origen gástrico o duodenal y el $85 \%$ es de origen esofágico; y debido a circunstancias anatómicas, es más frecuente en los $5 \mathrm{~cm}$ distales del mismo. En condiciones normales, estas venas se dirigen en forma longitudinal por la lámina propia de la mucosa esofágica; en la hipertensión portal, el aumento de la presión y del flujo sanguíneo incrementa su calibre y que las hace superficiales, por lo que pierden la protección ofrecida por los tejidos circundantes. Cuando esta presión supera los $12 \mathrm{~mm} \mathrm{Hg}$ de gradiente portovenoso, se aumenta el riesgo de ruptura varicosa con el consiguiente sangrado digestivo (2).

$\mathrm{Al}$ momento del ingreso, estos pacientes requieren una evaluación adecuada, estabilización respiratoria y hemodinámica (teniendo en cuenta el inicio de líquidos intravenosos y protección de la vía aérea), e incluso intubación orotraqueal en caso de deterioro del estado de conciencia que aumente el riesgo de broncoaspiración de material hemático. También se debe considerar el requerimiento de hemoderivados para mantener una hemoglobina entre 7 y $8 \mathrm{~g} / \mathrm{dL}$.

Así mismo, el uso de antibióticos es de relevancia en los pacientes cirróticos con hemorragia digestiva, ya que las complicaciones infecciosas en este grupo de pacientes es causante de morbimortalidad significativa. La recomendación del consenso de Baveno es el uso de ceftriaxona 1 $\mathrm{g} /$ día en poblaciones con alta resistencia a quinolinas o en quienes se ha usado profilaxis con este grupo de antibióticos previamente (1).

Debe realizarse la endoscopia digestiva con intención terapéutica lo más pronto posible ya que se ha identificado una correlación directa entre su demora mayor a 15 horas desde el ingreso y la mortalidad intrahospitalaria (3).

Sin embargo, al menos en el $10 \%$ de los casos no hay respuesta, por lo que se mantiene el sangrado activo a pesar de las medidas hemostáticas convencionales como lo pueden ser la ligadura con bandas y la inyección de soluciones esclerosantes utilizadas simultáneamente con vasodilatadores esplácnicos como la terlipresina, la somatostatina o el octreótido (4).

Aunque hay consenso en que las comunicaciones intrahepáticas transyugulares portosistémicas (TIPS) son una herramienta útil en el tratamiento de pacientes con sangrado de origen varicoso con cirrosis Child-Pugh C con más de 13 puntos, en aquellos con Child-Pugh B que tienen un sangrado activo al momento de la primera endoscopia y en aquellos pacientes con fallos en el tratamiento médico y endoscópico, no deja de ser una terapia con dificultades como lo pueden ser el empeoramiento clínico de los pacientes con insuficiencia hepática severa (lo que limitaría su uso a un pequeño grupo de pacientes en quienes se encontraría indicado) y la disponibilidad del recurso idealmente en las siguientes 24 horas luego del episodio de sangrado digestivo (por lo que un número importante de pacientes va a continuar sangrando, situación que ocasiona su muerte en cuestión de horas) $(5,6)$.

Por una parte, ha venido en aumento el uso de dispositivos hemostáticos temporales como el balón de SengstakenBlakemore o las prótesis metálicas autoexpandibles totalmente recubiertas (PMAR) como métodos hemostáticos que buscan el control del sangrado refractario mediante compresión sobre la várice sangrante; sin embargo, aunque el balón de Sengstaken-Blakemore se usa desde hace aproximadamente 25 años, ha demostrado serias dificultades en su colocación adecuada, por ser un procedimiento sin la posibilidad de una verificación de la localización de los balones, con la incomodidad para el paciente de necesitar un sistema de tracción que asegure la compresión sobre el fondo gástrico y el poco tiempo, muchas veces insuficiente (24 horas), para instaurar una terapia definitiva $(7,8)$.

Por otra parte, las PMAR son un método relativamente sencillo y disponible, que no requiere de la guía fluoroscópica y que cuenta con ventajas como el control del sangrado hasta del $100 \%$; sin morbimortalidad significativa asociada; el reinicio temprano de la vía oral y ser una solución temporal más prolongada (terapia puente), entre 7 y 14 días, lo que permite instaurar medidas para disminuir la presión portal, administrar antibióticos (8) y, posteriormente, llevar a los pacientes a tratamientos de más largo plazo como la colocación de TIPS, la realización de trasplante hepático o incluso el manejo con ligadura con bandas en los pacientes en los que inicialmente no fue posible realizarlo por una visualización limitada o inestabilidad hemodinámica del paciente (4). Si con estas medidas no se logra el control efectivo del sangrado, no habría tiempo de implementar otras, lo que en muchos casos causaría la muerte del paciente (9).

En la gran mayoría de los casos, las prótesis se retiran sin complicaciones luego de que el tratamiento definitivo se ha instaurado (9). Sin embargo, es claro que el riesgo de resangrado temprano luego del retiro es alto si no se ha llevado al paciente a un tratamiento definitivo y en este momento deben tenerse en cuenta las posibles contraindicaciones para dichos tratamientos como la alteración severa de la función hepática previa al sangrado en el caso de los TIPS (bilirrubinas $>3$, alteración en el tiempo de tromboplastina o la presencia previa de encefalopatía hepática) o la presencia de 
una neoplasia hepática central; y en el caso del trasplante, el consumo de alcohol en los últimos 6 meses previo al evento o la presencia de comorbilidades cardíacas, pulmonares, infecciosas o neoplásicas que lo contraindiquen $(4,10,11)$.

Aunque en esta condición específica no existen estudios aleatorizados y cegados (que serían el modelo para determinar si la intervención es adecuada), las dificultades metodológicas para llevar esto a cabo hacen prácticamente imposible la realización de este tipo de estudios teniendo en cuenta que es un dispositivo que no se puede cegar, el pronóstico del paciente con o sin intervención es malo a corto plazo y no hay un grupo control satisfactorio (6). Por esta razón, la experiencia plasmada en series de casos es la principal herramienta de reporte de esta intervención a nivel mundial y sus conclusiones han llegado a ser incluidas en el consenso de Baveno en su sexta edición, reunión llevada a cabo en 2015 como una terapia de salvamento para los pacientes con hemorragia varicosa refractaria (1).

\section{JUSTIFICACIÓN}

Un gran porcentaje de los pacientes con hemorragia varicosa refractaria a tratamientos convencionales mueren o tienen complicaciones serias derivadas de tratamientos invasivos como cirugía de urgencias, por lo que su manejo con prótesis metálicas autoexpandibles podría ser una medida efectiva para controlar el sangrado eficientemente, logrando aumentar la expectativa de vida de estos pacientes y llevar a algunos de ellos a terapias más definitivas como lo podría ser la colocación de TIPS o trasplante hepático.

En el momento no hay en nuestro medio experiencia reportada ni el ajuste de la técnica a nuestros recursos y necesidades.

\section{REFLEXIÓN}

En muchos países del mundo, se han utilizado las prótesis metálicas autoexpandibles como medidas de salvamento en pacientes con sangrado digestivo secundario a várices esofágicas que es refractario a las medidas endoscópicas convencionales como lo son la ligadura con bandas y la escleroterapia, con resultados contundentes en cuanto a control inmediato de sangrado que llega hasta el 96\% (12) y aumento de la expectativa de vida de los pacientes, permitiendo que sean llevados a tratamientos puente mientras son sometidos a terapias con intensión curativa como lo puede ser la terapia endoscópica de erradicación de várices o en mejor de los casos, el trasplante hepático. Los pacientes en quien no se logra el control inmediato del sangrado, son principalmente pacientes con várices gástricas o de la unión que no alcanzan a ser cubiertas por el cuerpo de la prótesis, requiriendo manejos complementarios como lo puede ser la inyección de cianoacrilato (13).

La mayoría de los estudios reportados se realizó con el dispositivo SX-Ella Danis stent (Ella-CS, Hradec Kralove, República Checa) (Figura 1), desarrollado específicamente para la colocación en pacientes con sangrado varicoso sin necesidad de asistencia endoscópica o fluoroscópica, ya que el sistema de colocación cuenta con un balón distal que se infla en la cámara gástrica y que permite que se ubique inmediatamente subcardial al ser halado, asegurando la colocación de la prótesis inmediatamente proximal al balón (esófago distal). Es una prótesis de nitinol totalmente recubierta de 13,5 $\mathrm{cm}$ de longitud, un diámetro en el cuerpo de $25 \mathrm{~mm}$ y copas de un tamaño entre 28 y $30 \mathrm{~mm}$; además, cuenta con 2 cintas metálicas de retiro ubicadas en los aspectos más proximales
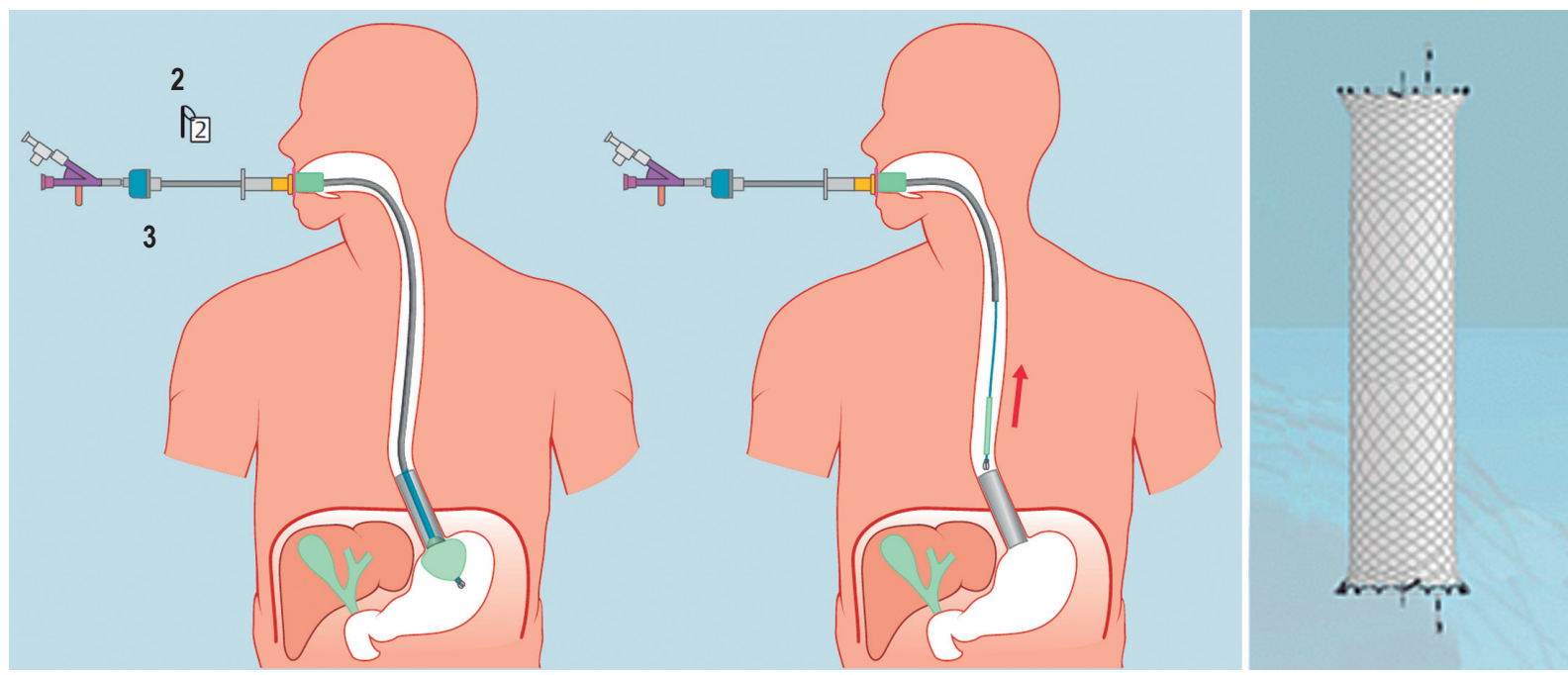

Figura 1. Sistema de colocación del dispositivo SX-Ella Danis stent (Ella-CS, Hradec Kralove, República Checa). 
y distales de la prótesis que permiten su reposicionamiento y extracción $(4,13,14)$ (Figura 2).

En los estudios realizados con este dispositivo, se ha reportado un tiempo de colocación de entre 4 y $10 \mathrm{~min}$ con una eficacia medida en el control del sangrado del $77 \%$ de los casos y una tasa de complicaciones inferior al $1 \%$ debido a la ulceración del esófago distal sin consecuencias clínicamente significativas (15).

Sin embargo, en instituciones donde se encuentre disponible el recurso endoscópico para el manejo de los pacientes con hemorragia digestiva, una posibilidad es la colocación de una prótesis autoexpandible con un sistema de colocación convencional, soportado por los estudios publicados por Hubmann (16) en los que se utilizó la prótesis Choo NES-T18-080-070, o el estudio de Dahlerup (17) en el que se usó la prótesis antirrefllujo SHIM, con unos resultados similares a los encontrados con la prótesis checa previamente descrita.

Para la colocación de este tipo de prótesis, previa medición de la posición de la unión gastroesofágica y del sitio del sangrado varicoso, se coloca la prótesis autoexpandible totalmente recubierta, idealmente con un diámetro del cuerpo $>25 \mathrm{~mm}$ y en la copas de 28 a $30 \mathrm{~mm}$, logrando un control adecuado del sangrado en más del $90 \%$ de los casos sin la necesidad de trasladar al paciente a salas de fluoroscopia y con las ventajas de estar disponibles en la mayoría de servicios de endoscopia y evidenciar endoscópicamente el control del sangrado; aunque se debe descartar el uso de este tipo de prótesis en sangrados derivados de várices gástricas.

Dentro de las complicaciones más frecuentes se encuentra la migración de la prótesis hacia el estómago, que puede ocurrir entre el $21 \%$ y el $36 \%$ de los casos, sin una correlación con un aumento de la incidencia de episodios de resangrado varicoso luego de la migración (13). Zakaria y colaboradores sugirieron que la realización de una endoscopia de confirmación de un adecuado posicionamiento de la prótesis 5 minutos después de la colocación favorece su completa expansión y disminuye el riesgo de migración (12). Otra complicación relativamente frecuente es la ulceración superficial principalmente en el aspecto más proximal de la prótesis, evidenciado en el momento de la extracción; sin embargo, no hay un desenlace patológico significativo de este hallazgo (12). En la literatura se han descrito otras complicaciones como la obstrucción de la vía aérea secundaria a compresión extrínseca, principalmente en pacientes con antecedentes de cirugía de reducción de volumen pulmonar por enfisema o alteraciones congénitas de la vía aérea (18).

En noviembre de 2015, Marot y colaboradores publicaron la primera revisión sistemática con metaanálisis que incluyó 13 estudios a nivel mundial que cumplían con los criterios de inclusión, estos estudios suman 146 pacientes de los que se obtuvieron datos relevantes dentro de la ponderación de la aplicación de la intervención. Se concluyó que la mortalidad a los 30 días era del $36 \%$, que aunque no es comparable con la de un grupo control, se asumió una disminución significativa con respecto a la mortalidad en pacientes sin la misma, que podría ser $>60 \%$. Las tasas de migración fueron del $28 \%$ y la utilidad como terapia puente de la intervención para trasplante fue del $10 \%$ y para la colocación de TIPS fue del $26 \%$ de los casos, cifras muy superiores a las documentadas en pacientes con sangrado varicoso refractario (6).

\section{CONCLUSIONES}

Las prótesis esofágicas autoexpandibles son herramientas al alcance del profesional de la salud en muchas instituciones y útiles en el manejo de pacientes con sangrado por várices esofágicas refractarias a medidas farmacológicas y endoscópicas convencionales.

El reporte de complicaciones, principalmente la migración o el resangrado, ha generado el desarrollo de dispositivos de colocación y prótesis diseñadas específicamente
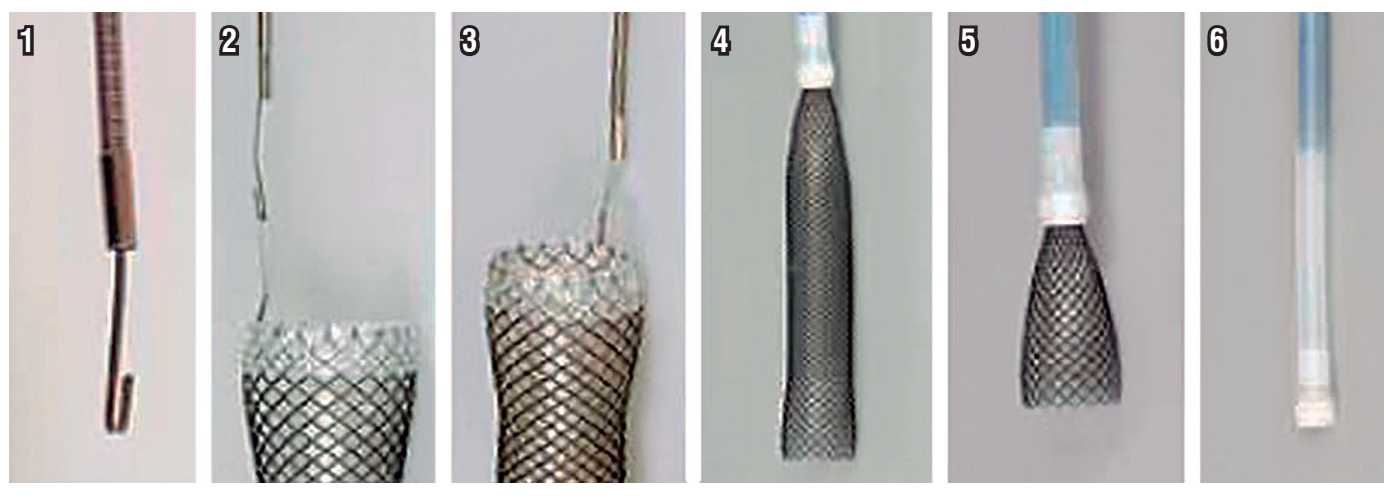

Figura 2. Sistema de retiro del dispositivo SX-Ella Danis stent (Ella-CS, Hradec Kralove, República Checa). 
para esta aplicación que pudiesen disminuir algunas limitaciones $y$, en especial, ser una respuesta para los servicios que no cuentan de modo permanente con la disponibilidad de tratamientos endoscópicos y requieren de la derivación del paciente a un nivel de atención superior previa estabilización e, idealmente, control de un sangrado profuso, muchas veces mortal a largo plazo.

\section{REFERENCIAS}

1. de Franchis R; Baveno VI Faculty. Expanding consensus in portal hypertension: Report of the Baveno VI Consensus Workshop: Stratifying risk and individualizing care for portal hypertension. J Hepatol. 2015;63(3):743-52. doi: 10.1016/j.jhep.2015.05.022.

2. Arakawa M, Masuzaki T, Okuda K. Pathology of fundic varices of the stomach and rupture. J Gastroenterol Hepatol. 2002; 17(10):1064-9.

3. Hsu YC, Chung CS, Tseng $\mathrm{CH}$, et al. Delayed endoscopy as a risk factor for in-hospital mortality in cirrhotic patients with acute variceal hemorrhage. J Gastroenterol Hepatol. 2009;24(7):1294-9. doi: 10.1111/j.14401746.2009.05903.x.

4. Dechêne A, El Fouly AH, Bechmann LP, et al. Acute management of refractory variceal bleeding in liver cirrhosis by self-expanding metal stents. Digestion. 2012;85(3):185-91. doi: $10.1159 / 000335081$.

5. Escorsell A, Bosch J. Self-expandable metal stents in the treatment of acute esophageal variceal bleeding. Gastroenterol Res Pract. 2011;2011:910986. doi: 10.1155/2011/910986.

6. Marot A, Trépo E, Doerig C, et al. Systematic review with meta-analysis: self-expanding metal stents in patients with cirrhosis and severe or refractory oesophageal variceal bleeding. Aliment Pharmacol Ther. 2015;42(11-12):1250-60. doi: 10.1111 /apt.13424.

7. Maufa F, Al-Kawas FH. Role of self-expandable metal stents in acute variceal bleeding. Int J Hepatol. 2012;2012:418369. doi: $10.1155 / 2012 / 418369$.

8. El Sayed G, Tarff S, O’Beirne J, et al. Endoscopy management algorithms: role of cyanoacrylate glue injection and self-expanding metal stents in acute variceal haemorrhage. Frontline Gastroenterol. 2015;6(3):208-216. doi: 10.1136/ flgastro-2013-100428.
9. Müller M, Seufferlein T, Perkhofer L, et al. Self-expandable metal stents for persisting esophageal variceal bleeding after band ligation or injection-therapy: A retrospective study. PLoS One. 2015;10(6):e0126525. doi: 10.1371/journal. pone.0126525.

10. García-Pagán JC, Caca K, Bureau C, et al. Early use of TIPS in patients with cirrhosis and variceal bleeding. $\mathrm{N}$ Engl J Med. 2010;362(25):2370-9. doi: 10.1056/NEJMoa0910102.

11. Deltenre P, Trépo E, Rudler M, et al. Early transjugular intrahepatic portosystemic shunt in cirrhotic patients with acute variceal bleeding: a systematic review and metaanalysis of controlled trials. Eur J Gastroenterol Hepatol. 2015;27(9):e1-9. doi: 10.1097/MEG.0000000000000403.

12. Zakaria MS, Hamza IM, Mohey MA, et al. The first Egyptian experience using new self-expandable metal stents in acute esophageal variceal bleeding: pilot study. Saudi J Gastroenterol. 2013;19(4):177-81. doi: 10.4103/13193767.114516.

13. Changela K, Ona MA, Anand S, et al. Self-Expanding Metal Stent (SEMS): an innovative rescue therapy for refractory acute variceal bleeding. Endosc Int Open. 2014;2(4):E24451. doi: 10.1055/s-0034-1377980.

14. Hogan B, Patch D, Burroughs A, et al. Use of the SX-Ella selfexpanding mesh metal stent in the management of complex variceal haemorrhage: initial experience in a single centre. J Hepatol. 2009;50(Suppl 1):s86-7. doi: 10.1016/S01688278(09)60214-2.

15. Pontone S, Giusto M, Filippini A, et al. Hemostasis in uncontrolled esophageal variceal bleeding by self-expanding metal stents: a systematic review. Gastroenterol Hepatol Bed Bench. 2016;9(1):6-11.

16. Hubmann R, Bodlaj G, Czompo M, et al. The use of selfexpanding metal stents to treat acute esophageal variceal bleeding. Endoscopy. 2006;38(9):896-901. doi: 10.1055/s2006-944662.

17. Dahlerup JF, Kruse A, Grønbaek H, et al. Therapy of continuously bleeding oesophageal varices by self expanding metal stents. Ugeskr Laeger. 2007;169(34):2784-5.

18. Dechene A, Adamzik M, Gerken G, et al. Acute bronchial obstruction following esophageal stent implantation for variceal bleeding. Endoscopy. 2009;41 Suppl 2:E146-7. doi: $10.1055 / \mathrm{s}-0028-1119725$. 